\title{
Renal Pyramid
}

National Cancer Institute

\section{Source}

National Cancer Institute. Renal Pyramid. NCI Thesaurus. Code C12886.

A cone-shaped structure of the kidney. It is located in the medullary portion of the kidney (pyramid of Malpighi) or the cortical portion of the kidney (pyramid of Ferrein). 